\title{
Acute Care for Elders (ACE) Team Model of Care: A Clinical Overview
}

\author{
Kellie L. Flood ${ }^{1,2, *}$, Katrina Booth ${ }^{1,2,3}$, Jasmine Vickers ${ }^{1}$, Emily Simmons ${ }^{2}$, David H. James 2 (1), \\ Shari Biswal ${ }^{2}$, Jill Deaver ${ }^{4}$, Marjorie Lee White ${ }^{2,5}$ and Ella H. Bowman 1,3 (1) \\ 1 Division of Gerontology, Geriatrics, and Palliative Care, University of Alabama, \\ Birmingham, AL 35294, USA; kjulian@uabmc.edu (K.B.); jkvicker@uab.edu (J.V.); \\ ehbowman@uabmc.edu (E.H.B.) \\ 2 University of Alabama at Birmingham Hospital, Birmingham, AL 35294, USA; esimmons@uabmc.edu (E.S.); \\ djames@uabmc.edu (D.H.J.); sbiswal@uabmc.edu (S.B.); mlwhite@uab.edu (M.L.W.) \\ 3 Birmingham Veterans Affairs Medical Center, Birmingham, AL 35294, USA \\ 4 Lister Hill Library of the Health Sciences, UAB Libraries, University of Alabama, \\ Birmingham, AL 35294, USA; jilld@uab.edu \\ 5 Division of Pediatric Emergency Medicine, University of Alabama, Birmingham, AL 35294, USA \\ * Correspondence: kflood@uabmc.edu; Tel.: +1-205-934-9261
}

Received: 2 July 2018; Accepted: 2 August 2018; Published: 6 August 2018

\begin{abstract}
The Institute of Medicine (IOM) Reports of To Err is Human and Crossing the Quality Chasm have called for more interprofessional and coordinated hospital care. For over 20 years, Acute Care for Elders (ACE) Units and models of care that disseminate ACE principles have demonstrated outcomes in-line with the IOM goals. The objective of this overview is to provide a concise summary of studies that describe outcomes of ACE models of care published in 1995 or later. Twenty-two studies met the inclusion. Of these, 19 studies were from ACE Units and three were evaluations of ACE Services, or teams that cared for patients on more than one hospital unit. Outcomes from these studies included increased adherence to evidence-based geriatric care processes, improved patient functional status at time of hospital discharge, and reductions in length of stay and costs in patients admitted to ACE models compared to usual care. These outcomes represent value-based care. As interprofessional team models are adopted, training in successful team functioning will also be needed.
\end{abstract}

Keywords: Acute Care for Elders; ACE Unit; interprofessional team; interdisciplinary

\section{Introduction}

The launching point for the quality movement in healthcare was the 1999 Institute of Medicine (IOM) Report To Err is Human, which concluded tens of thousands of Americans die annually from preventable errors in care [1]. In 2001, the follow-up report Crossing the Quality Chasm further described the gap between evidence-based healthcare and care delivered. This 2001 report acknowledged an aging population and thus, an increasing proportion of patients with multiple chronic conditions. The report also called for fundamental redesign of care delivery to include systems to train and support high performing patient-centered teams [2].

Forward thinking geriatric clinicians recognized the need for interprofessional team models of acute care prior to these IOM reports. The Acute Care for Elders (ACE) Unit is one of the better recognized examples. The ACE Unit model consists of several core components: (1) patientcentered care with proactive geriatric assessments, (2) nurse-driven care plans for the prevention and management of geriatric syndromes, (3) comprehensive care transition planning beginning 
at admission, and (4) medical care review with the goal to prevent iatrogenesis and incident geriatric syndromes. This care is delivered by an interprofessional team that conducts frequent (usually Monday-Friday) team meetings (ACE rounds) to develop the geriatric care plans for each patient. In most ACE Units a geriatrician or geriatric advanced nurse practitioner either participates in the team rounds and/or is an attending practitioner for patients. In addition, ideally the physical environment of the ACE Unit is modified to promote safe mobility and cognitive stimulation [3,4].

The first randomized controlled trial (RCT) of an ACE Unit was published in 1995 by Landefeld et al., and demonstrated the benefits of this care delivery redesign [4]. This publication was a seminal moment, launching ACE Unit development and research that continues today. With this in mind, we present here an overview of studies evaluating outcomes from ACE models and published in 1995 or later. We provide this overview with two primary objectives: (1) to provide a concise reference tool for use by clinicians and researchers working in the field of ACE team models of care, and (2) to assist in identifying opportunities for future ACE efforts, including scaling and dissemination strategies and future quality and research efforts.

\section{Methods}

Utilizing published criteria for fourteen recognized types of literature reviews, we present here a literature "overview," defined as "any summary of the medical literature that attempts to survey the literature and describe its characteristics." Overviews "can provide a broad and often comprehensive summation of a topic area" and the analysis may be "chronological, conceptual, or thematic" [5]. To complete this overview, we conducted a literature search using Pubmed, Embase, CINAHL, and Scopus with the following database-specific subject headings, key terms, and phrases: "Acute Care"; "Acute Care for Elders"; "Interprofessional Teams"; "Interdisciplinary Teams"; "Interprofessional Relations"; "Geriatrics"; "Aged"; "Aged 80 and older"; and "Elderly". After removing duplicates, two authors (KLF, KB) reviewed all titles and abstracts for studies satisfying our inclusion/exclusion criteria to ensure adherence to the ACE model (Table 1). This was followed by critical reading by four authors (KLF, KB, JV, DJ) of full text articles if insufficient information was provided in the title or abstract to determine relevance. Additionally, the reference lists of included retrieved systematic reviews and meta-analyses were also examined for other articles that would meet the inclusion criteria for this overview (Figure 1). In our final list of relevant articles, we did include one study from an ACE Unit accepting patients with a younger age threshold than traditional ACE units (60 years and over) [6].

Table 1. Study inclusion and exclusion criteria.

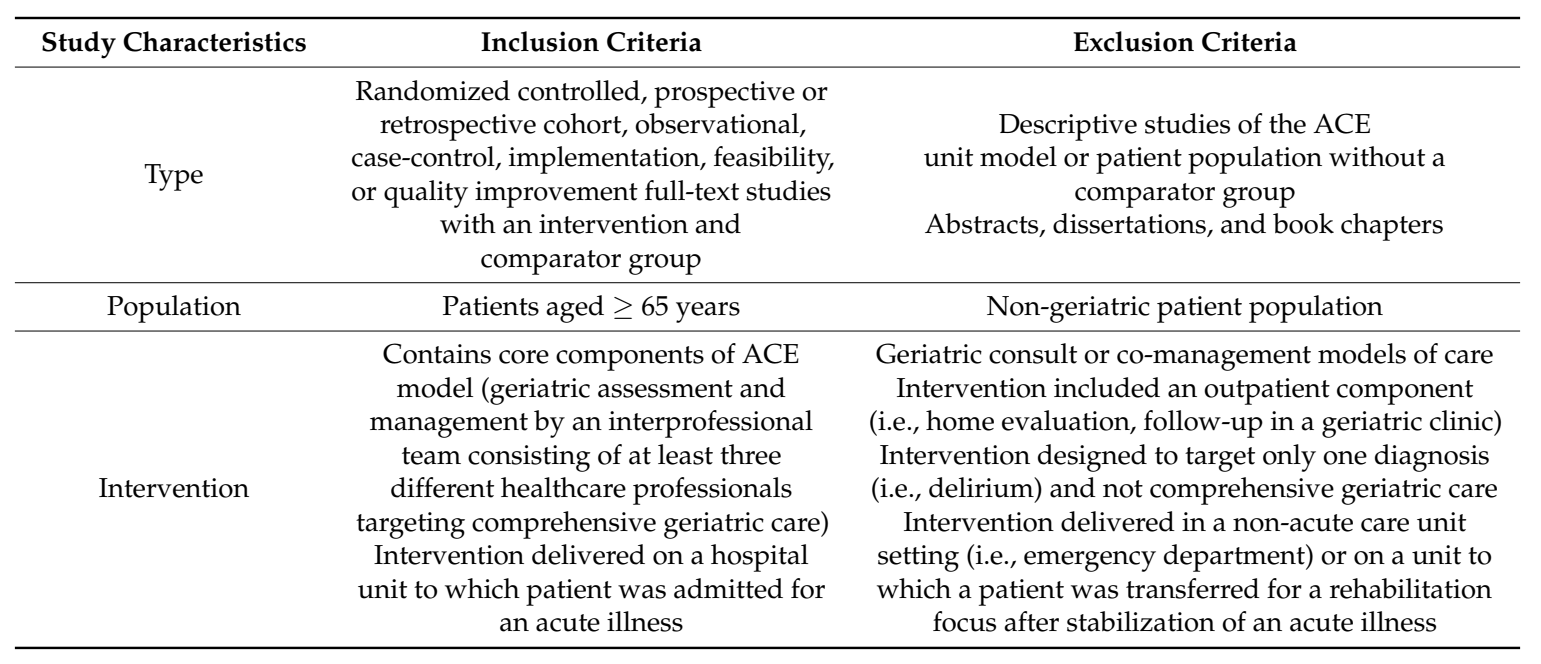


Table 1. Cont.

\begin{tabular}{ccc}
\hline Study Characteristics & Inclusion Criteria & Exclusion Criteria \\
\hline Comparator Group & $\begin{array}{c}\text { Usual/routine hospital care in a similar } \\
\text { patient population on the same or } \\
\text { similar type of unit or service }\end{array}$ & No comparator or usual/outline care group \\
\hline Outcome Measures & $\begin{array}{c}\text { Patient related geriatric care process, } \\
\text { clinical, satisfaction, quality of life, or } \\
\text { healthcare utilization outcomes }\end{array}$ & Staff or provider educational or perception outcomes \\
\hline Language & English & Non-English \\
\hline Publication date & 1995 or later & Prior to 1995 \\
\hline
\end{tabular}

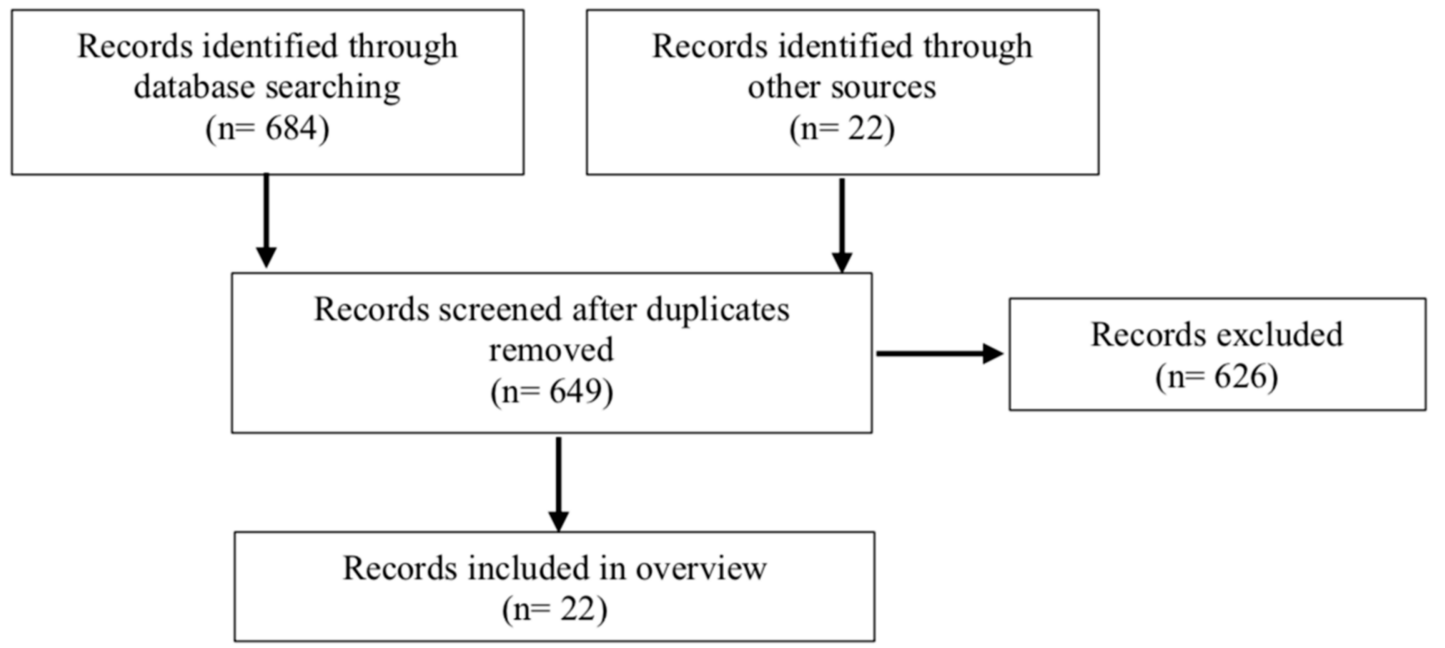

Figure 1. Literature review flow diagram.

\section{Results}

Our search strategy yielded twenty-two articles included in this overview. We present here the key findings from these studies in two categories of ACE models: (1) ACE Units, in which the care occurs for patients admitted to one geographically distinct hospital unit (19 studies), and (2) ACE Services, in which the team care is delivered by an admitting service caring for patients on more than one hospital unit ( 3 studies). Tables 2 and 3 are summaries of the key study characteristics and outcomes included in this overview. 
Table 2. ACE unit studies and key findings *.

\begin{tabular}{|c|c|c|c|c|c|c|c|}
\hline Study (Setting) & Design & $\begin{array}{l}\text { Patient Population (Mean } \\
\text { Age of ACE Cohort) }\end{array}$ & $\begin{array}{c}\text { ACE vs. UC } \\
\text { Attending Physicians } \\
\text { (Sample Sizes) }\end{array}$ & $\begin{array}{c}\text { Primary Outcome } \\
\text { Measured (ACE vs. UC) }\end{array}$ & $\begin{array}{l}\text { Secondary Outcome(s) Measured } \\
\text { (ACE vs. UC) }\end{array}$ & $\begin{array}{l}\text { Study and/or } \\
\text { Intervention } \\
\text { Limitations }\end{array}$ & $\begin{array}{c}\text { Study Strengths } \\
\text { and/or Intervention } \\
\text { Innovations }\end{array}$ \\
\hline $\begin{array}{l}\text { Landefeld et al., } 1995 \text { [4] } \\
\text { (university hospital, USA) }\end{array}$ & RCT & $\begin{array}{l}\text { General medical patients } \\
\text { aged } \geq 70 \text { years }(80.2)\end{array}$ & $\begin{array}{l}\text { Internists on ACE } \\
\text { (327) and UC (324) } \\
\text { units }\end{array}$ & $\begin{array}{l}\text { Significantly improved ADL } \\
\text { performance from baseline } \\
(p=0.05) \text { and admission } \\
(p=0.009) \text { to discharge }\end{array}$ & $\begin{array}{c}\text { Significantly reduced PACF } \\
\text { placement }(14 \% \text { vs. } 22 \%, p=0.01) \\
\text { Significantly improved overall } \\
\text { health status }(p<0.001) \\
\text { at discharge } \\
\text { No difference in hospital charges }\end{array}$ & $\begin{array}{l}\text { Utilized charges for } \\
\text { cost analysis }\end{array}$ & $\begin{array}{l}\text { Randomized } \\
\text { Improved outcomes in } \\
\text { subgroup and } \\
\text { multivariate analyses }\end{array}$ \\
\hline $\begin{array}{c}\text { Covinsky et al., 1997 [7] } \\
\text { (university hospital, USA) }\end{array}$ & $\begin{array}{l}\text { RCT; Cost analysis } \\
\text { from Landefeld et al., } \\
\text { study }\end{array}$ & $\begin{array}{l}\text { General medical patients } \\
\text { aged } \geq 70 \text { years }(80.7)\end{array}$ & $\begin{array}{l}\text { Internists on ACE } \\
\text { (326) and UC (324) } \\
\text { units }\end{array}$ & $\begin{array}{l}\text { No significant reduction in } \\
\text { total costs per case }(\$ 6608 \\
\text { vs. } \$ 7240, p=0.93)\end{array}$ & $\begin{array}{l}\text { No significant reduction in LOS } \\
\quad(7.5 \text { vs. } 8.4 \text { days, } p=0.449) \\
\text { Significantly reduced } 90 \text {-day PACF } \\
\text { use }(24.1 \% \text { vs. } 32.3 \%, p=0.034)\end{array}$ & $\begin{array}{l}\text { Total costs includes } \\
\text { indirect costs } \\
\text { Lacked power to } \\
\text { determine significance } \\
\text { in cost difference }\end{array}$ & $\begin{array}{c}\text { Randomized } \\
\text { Included ACE start-up } \\
\text { costs, likely } \\
\text { under-estimating } \\
\text { long-term cost savings }\end{array}$ \\
\hline $\begin{array}{c}\text { Stewart et al., } 1999 \text { [8] } \\
\text { (community teaching } \\
\text { hospital, USA) }\end{array}$ & $\begin{array}{c}\text { Prospective } \\
\text { observational }\end{array}$ & $\begin{array}{c}\text { General medical or surgical } \\
\text { patients } \\
\text { aged } \geq 75 \text { years (86) }\end{array}$ & $\begin{array}{l}\text { Internist or Surgeon } \\
\text { on ACE (34) and UC } \\
\text { (27) units }\end{array}$ & $\begin{array}{l}\text { No significant difference in } \\
\text { LOS (6.0 vs. 7.1 days, } \\
p=0.06)\end{array}$ & $\begin{array}{l}\text { Significantly reduced charges } \\
(\$ 6,223 \text { vs. } \$ 10,042, p<0.01)\end{array}$ & $\begin{array}{l}\text { Non-randomized } \\
\text { Multiple significantly } \\
\text { different baseline } \\
\text { characteristics } \\
\text { between cohorts and } \\
\text { results unadjusted } \\
\text { Utilized charges for } \\
\text { cost analysis }\end{array}$ & $\begin{array}{l}\text { ACE care for medical } \\
\text { and surgical patients }\end{array}$ \\
\hline $\begin{array}{l}\text { Counsell et al., 2000 [9] } \\
\text { (community teaching } \\
\text { hospital, USA) }\end{array}$ & RCT & $\begin{array}{l}\text { Community-dwelling } \\
\text { general medical patients } \\
\text { with LOS } \geq 2 \text { days } \\
\text { aged } \geq 70 \text { years }(80)\end{array}$ & $\begin{array}{l}\text { Internist or Family } \\
\text { Practice attending on } \\
\text { ACE (767) and UC } \\
\text { (764) units }\end{array}$ & $\begin{array}{l}\text { No significant difference in } \\
\text { ADL performance at } \\
\text { discharge } \\
\text { Significant reduction in } \\
\text { composite outcome (ADL } \\
\text { decline or PACF placement; } \\
34 \% \text { vs. } 40 \%, p=0.027 \text { ) }\end{array}$ & $\begin{array}{c}\text { Significant increased use of nursing } \\
\text { care plans }(79 \% \text { vs. } 50 \% ; p=0.001) \text {, } \\
\text { SW consults } \\
(50 \% \text { vs. } 43 \%, p=0.012) \text {, and PT } \\
\text { consults }(42 \% \text { vs. } 36 \%, p=0.027) \\
\text { Significant reduction in restraint } \\
\text { use ( } 2 \% \text { vs. } 6 \% ; p=0.001) \\
\text { Improved patient, caregiver, and } \\
\text { provider satisfaction }\end{array}$ & $\begin{array}{l}\text { ADL outcome may } \\
\text { have been influenced } \\
\text { by healthier patient } \\
\text { population and } \\
\text { shorter LOS than } \\
\text { Landefeld et al., RCT }\end{array}$ & $\begin{array}{l}\text { Randomized } \\
\text { Large sample size } \\
\text { Studied ACE in } \\
\text { patients not on a } \\
\text { teaching service }\end{array}$ \\
\hline $\begin{array}{l}\text { Asplund et al., } 2000 \text { [10] } \\
\text { (university hospital, } \\
\text { Sweden) }\end{array}$ & RCT & $\begin{array}{l}\text { General medical patients } \\
\text { aged } \geq 70 \text { years }(80.9)\end{array}$ & $\begin{array}{l}\text { Internist initially } \\
\text { followed by } \\
\text { Geriatrician on ACE } \\
\text { (190) vs. Internist on } \\
\text { UC (223) units }\end{array}$ & $\begin{array}{l}\text { No significant difference in } \\
\text { poor global outcome } \\
\text { measure } \alpha 3 \text { months } \\
\text { post-discharge (RR 1.06; } \\
95 \% \text { CI 0.84-1.34) }\end{array}$ & $\begin{array}{c}\text { Significantly reduced LOS } \\
(5.9 \text { vs. } 7.3 \text { days, } p=0.002) \\
\text { No difference in readmissions or } \\
\text { healthcare utilization at } 3 \text { months } \\
\text { No significant difference in hospital } \\
\text { mortality ( } 4 \% \text { vs. } 3 \%)\end{array}$ & $\begin{array}{l}\text { Per-protocol analysis } \\
\text { performed since } \\
\text { majority of ineligible } \\
\text { patients were due to } \\
\text { inappropriate } \\
\text { randomization } \\
\text { processes } \\
\end{array}$ & $\begin{array}{l}\text { Randomized } \\
\text { Evaluated function } \\
\text { and well-being } \\
\text { post-discharge }\end{array}$ \\
\hline $\begin{array}{l}\text { Salvedt et al., } 2002 \text { [11] } \\
\text { (university hospital, } \\
\text { Norway) }\end{array}$ & RCT & $\begin{array}{l}\text { General medical patients } \\
\text { meeting frailty criteria aged } \\
\geq 75 \text { years (81.8) }\end{array}$ & $\begin{array}{l}\text { Geriatrician on ACE } \\
\text { (127) vs. Internist or } \\
\text { Medical Subspecialists } \\
\text { on UC (127) units }\end{array}$ & $\begin{array}{c}\text { Significantly reduced } \\
\text { mortality at } 3(12 \% \text { vs. } 27 \% \text {, } \\
p=0.004) \text { and } 6 \text { months } \\
(16 \% \text { vs. } 29 \%, p=0.02) \\
\text { post-discharge }\end{array}$ & $\begin{array}{l}\text { Significantly increased LOS (15 vs. } \\
7 \text { days, } p<0.001) \\
\text { Significantly more ACE patients } \\
\text { with dementia, depression, and } \\
\text { delirium diagnoses documented } \\
(38 \% \text { vs. } 7 \%, p<0.001)\end{array}$ & $\begin{array}{l}\text { Non-USA based study } \\
\text { may have } \\
\text { influenced LOS } \\
\text { Some ACE patients } \\
\text { were transferred from } \\
\text { other units }\end{array}$ & $\begin{array}{l}\text { Randomized } \\
\text { Targeted frail patients } \\
\text { First to evaluated } \\
\text { mortality as a primary } \\
\text { outcome }\end{array}$ \\
\hline
\end{tabular}


Table 2. Cont.

\begin{tabular}{|c|c|c|c|c|c|c|c|}
\hline Study (Setting) & Design & $\begin{array}{l}\text { Patient Population (Mean } \\
\text { Age of ACE Cohort) }\end{array}$ & $\begin{array}{c}\text { ACE vs. UC } \\
\text { Attending Physicians } \\
\text { (Sample Sizes) }\end{array}$ & $\begin{array}{c}\text { Primary Outcome } \\
\text { Measured (ACE vs. UC) }\end{array}$ & $\begin{array}{c}\text { Secondary Outcome(s) Measured } \\
\text { (ACE vs. UC) }\end{array}$ & $\begin{array}{l}\text { Study and/or } \\
\text { Intervention } \\
\text { Limitations }\end{array}$ & $\begin{array}{c}\text { Study Strengths } \\
\text { and/or Intervention } \\
\text { Innovations }\end{array}$ \\
\hline $\begin{array}{c}\text { Naglie et al., } 2002 \text { [12] } \\
\text { (university } \\
\text { hospital, Canada) }\end{array}$ & RCT & $\begin{array}{l}\text { Patients with surgical hip } \\
\text { fracture repair } \\
\text { aged } \geq 70 \text { years }(83.8)\end{array}$ & $\begin{array}{l}\text { Geriatrician directed } \\
\text { medical care on } \\
\text { Ortho-ACE (141) vs. } \\
\text { Internist directed } \\
\text { medical care on } \\
\text { Ortho-UC (138) units }\end{array}$ & $\begin{array}{l}\text { No significant difference in } \\
\text { composite outcome } \\
\text { (\% patients alive with no } \\
\text { decline from baseline in } \\
\text { ambulation, transfers, or } \\
\text { place of residence } 6 \text { month } \\
\text { post-surgery; ; adjusted OR } \\
1.1,95 \% \text { CI } 0.6-2.1 \text { ) }\end{array}$ & $\begin{array}{l}\text { Significant increase in } \% \text { patients } \\
\text { with composite outcome (alive } \\
\text { with no decline from baseline in } \\
\text { ambulation, transfers, or place of } \\
\text { residence at } 6 \text { months) in analysis } \\
\text { of cognitively impaired patients } \\
\text { (47\% vs. } 24 \%, p=0.03) \\
\text { Significantly increased LOS } \\
(29.2 \text { vs. } 20.9 \text { days, } p<0.001)\end{array}$ & $\begin{array}{c}\text { Non-USA based study } \\
\text { may have } \\
\text { influenced LOS } \\
\text { Did not evaluate } \\
\text { in-hospital outcomes }\end{array}$ & $\begin{array}{l}\text { Randomized design } \\
\text { Extension of ACE to } \\
\text { orthopedic } \\
\text { surgery patients } \\
\text { Twice weekly } \\
\text { ACE rounds }\end{array}$ \\
\hline $\begin{array}{c}\text { Allen et al, } 2003 \text { [13] } \\
\text { (community teaching } \\
\text { hospital, USA) }\end{array}$ & Pre/post comparison & $\begin{array}{l}\text { Acute stroke patients } \\
\text { pre/post launch of } \\
\text { ACE-like stroke unit; no age } \\
\text { criteria reported (72) }\end{array}$ & $\begin{array}{l}\text { Neurologist 1-year pre } \\
(622) \text { vs. 1-year post } \\
(544) \text { stroke unit } \\
\text { utilizing ACE model }\end{array}$ & $\begin{array}{l}\text { Significantly reduced LOS } \\
(3.8 \text { vs. } 4.6 \text { days, } p<0.0001)\end{array}$ & $\begin{array}{c}\text { Significantly more patients } \\
\text { discharged home } \\
(62 \% \text { vs. } 50 \%, p<0.0001) \\
\text { Significantly increased proportion } \\
\text { of patients without a readmission } \\
\text { at 1-year }(41 \% \text { vs. } 18 \%, p<0.0001) \\
\text { Significantly reduced health system } \\
\text { Medicare stroke-specific and } \\
\text { risk-adjusted inpatient mortality } \\
(11.4 \% \text { vs. } 8.4 \%, p=0.02) \\
\text { No significant difference in } \\
\text { all-cause mortality } \\
(7 \% \text { vs. } 16 \% ; p=0.11)\end{array}$ & $\begin{array}{l}\text { Non-randomized } \\
\text { Data from } \\
\text { administrative } \\
\text { database }\end{array}$ & $\begin{array}{l}\text { ACE team model and } \\
\text { processes used to } \\
\text { develop an acute } \\
\text { stroke unit }\end{array}$ \\
\hline $\begin{array}{c}\text { Jayadevappa et al., } 2006 \\
\text { [14] (university } \\
\text { hospital, USA) }\end{array}$ & $\begin{array}{l}\text { Retrospective } \\
\text { case-control }\end{array}$ & $\begin{array}{l}\text { General medical patients } \\
\text { admitted for CHF, UTI, or } \\
\text { pneumonia } \\
\text { aged } \geq 65 \text { years }(79.6)\end{array}$ & $\begin{array}{l}\text { Internist or } \\
\text { Geriatrician on ACE } \\
\text { (680) vs. Internist on } \\
\text { UC (680) units }\end{array}$ & $\begin{array}{l}\text { Significantly reduced LOS } \\
\quad(4.9 \text { vs. } 5.9, p=0.01) \\
\text { Significantly reduced mean } \\
\text { costs }(\$ 13,586 \text { vs. } \$ 15,039, p \\
=0.012)\end{array}$ & $\begin{array}{l}\text { Reduced annual readmissions after } \\
\text { controlling for age, race, } \\
\text { comorbidities, and number of prior } \\
\text { admissions }\end{array}$ & $\begin{array}{l}\text { Non-randomized } \\
\text { Data from } \\
\text { administrative } \\
\text { database } \\
\text { Costs estimated from } \\
\text { a cost-to-charge ratio }\end{array}$ & $\begin{array}{l}\text { Adjusted for prior } \\
\text { admissions in } \\
\text { analyzing } \\
\text { readmission rate }\end{array}$ \\
\hline $\begin{array}{l}\text { Zelada et al., } 2009 \text { [15] } \\
\text { (military teaching } \\
\text { hospital, Peru) }\end{array}$ & $\begin{array}{l}\text { Prospective } \\
\text { observational }\end{array}$ & $\begin{array}{l}\text { General medical patients } \\
\text { aged } \geq 65 \text { years }(79.6)\end{array}$ & $\begin{array}{l}\text { Geriatrician on ACE } \\
\text { (68) vs. Internist on } \\
\text { UC (75) units }\end{array}$ & $\begin{array}{l}\text { Significantly reduced ADL } \\
\text { decline during } \\
\text { hospitalization } \\
(19 \% \text { vs. } 40 \%, p=0.013)\end{array}$ & $\begin{array}{l}\text { Increased OR for ADL decline in } \\
\text { UC patients ( } 4.24 ; 95 \% \text { CI 1.50-11.9) } \\
\text { Reduced LOS on ACE } \\
\text { (7.5 vs. } 9.92 \text { days, } p=0.03)\end{array}$ & $\begin{array}{l}\text { Non-randomized } \\
\text { Multiple significantly } \\
\text { different baseline } \\
\text { characteristics } \\
\text { between cohorts }\end{array}$ & $\begin{array}{l}\text { Once weekly } \\
\text { ACE rounds }\end{array}$ \\
\hline $\begin{array}{c}\text { Malone et al., } 2010 \text { [16] } \\
\text { (community } \\
\text { hospital, USA) }\end{array}$ & Pre/post comparison & $\begin{array}{l}\text { General medical or } \\
\text { urology patients } \\
\text { aged } \geq 65 \text { years } \\
\text { (no mean age reported) }\end{array}$ & $\begin{array}{l}\text { Urology or Internist } \\
\text { on medical-surgical } \\
\text { units pre (478) vs. post } \\
(406) \text { e-Geriatrician }\end{array}$ & $\begin{array}{l}\text { Significantly reduced use of } \\
\text { urinary catheters } \\
(26.2 \text { vs. } 20.1 \%, p=0.03) \\
\text { Significantly increased } \\
\text { physical therapy referrals } \\
(27.0 \% \text { vs. } 39.1 \% ; p<0.001)\end{array}$ & $\begin{array}{l}\text { No significant difference in use of } \\
\text { physical restraints, social service } \\
\text { assessments, high-risk medications, } \\
\text { LOS, or 30-day readmissions }\end{array}$ & $\begin{array}{l}\text { Non-randomized } \\
\text { No formal tracking } \\
\text { of whether } \\
\text { recommendations } \\
\text { made by geriatricians } \\
\text { are followed }\end{array}$ & $\begin{array}{l}\text { Use of EMR tool to } \\
\text { disseminate ACE care } \\
\text { Twice weekly } \\
\text { e-Geriatrician in } \\
\text { ACE rounds }\end{array}$ \\
\hline
\end{tabular}


Table 2. Cont.

\begin{tabular}{|c|c|c|c|c|c|c|c|}
\hline Study (Setting) & Design & $\begin{array}{l}\text { Patient Population (Mean } \\
\text { Age of ACE Cohort) }\end{array}$ & $\begin{array}{c}\text { ACE vs. UC } \\
\text { Attending Physicians } \\
\text { (Sample Sizes) }\end{array}$ & $\begin{array}{c}\text { Primary Outcome } \\
\text { Measured (ACE vs. UC) }\end{array}$ & $\begin{array}{l}\text { Secondary Outcome(s) Measured } \\
\text { (ACE vs. UC) }\end{array}$ & $\begin{array}{l}\text { Study and/or } \\
\text { Intervention } \\
\text { Limitations }\end{array}$ & $\begin{array}{l}\text { Study Strengths } \\
\text { and/or Intervention } \\
\text { Innovations }\end{array}$ \\
\hline $\begin{array}{c}\text { Flood et al., } 2011 \text { [17] } \\
\text { (university hospital, USA) }\end{array}$ & $\begin{array}{l}\text { Retrospective chart } \\
\text { review }\end{array}$ & $\begin{array}{c}\text { Hematology-oncology } \\
\text { patients with } \\
\text { nutritional deficits } \\
\text { aged } \geq 65 \text { years }(75.25)\end{array}$ & $\begin{array}{l}\text { Private Oncologist or } \\
\text { Teaching attending } \\
\text { with residents on } \\
\text { Oncology-ACE (103) } \\
\text { vs. UC (82) units }\end{array}$ & $\begin{array}{l}\text { Significantly increased OR } \\
\text { for receiving a formal } \\
\text { nutrition consult } \\
(2.1,95 \% \text { CI 1.033-4.300) } \\
\text { and nutritional } \\
\text { supplements ordered } \\
\text { (2.5; } 95 \% \text { CI 1.221-5.319) } \\
\text { in adjusted analysis }\end{array}$ & $\begin{array}{l}\text { Significantly increased proportion } \\
\text { of patients receiving a nutrition } \\
\text { consult }(63.1 \% \text { vs. } 45.1 \%, p=0.011) \\
\text { and an order for supplements } \\
(57.3 \% \text { vs. } 32.9 \%, p=0.001) \\
\text { in unadjusted analysis }\end{array}$ & $\begin{array}{c}\text { Non-randomized } \\
\text { No standardized } \\
\text { nutritional risk } \\
\text { screening process } \\
\text { on units } \\
\text { Significantly more } \\
\text { OACE cohort with } \\
\text { low BMI } \\
\text { No clinical } \\
\text { outcomes measured }\end{array}$ & $\begin{array}{l}\text { Extension of ACE } \\
\text { model to hematology/ } \\
\text { oncology patients } \\
\text { Evaluated role of ACE } \\
\text { on nutritional } \\
\text { processes of care }\end{array}$ \\
\hline $\begin{array}{c}\text { Barnes et al., } 2012 \text { [18] } \\
\text { (university hospital, USA) }\end{array}$ & $\begin{array}{l}\text { RCT (2nd RCT } \\
\text { from same ACE unit in } \\
\text { Landefeld et al., study) }\end{array}$ & $\begin{array}{l}\text { Community-dwelling } \\
\text { general medical patients } \\
\text { aged } \geq 70 \text { years }(81)\end{array}$ & $\begin{array}{l}\text { Internists on ACE } \\
(858) \text { and UC ( } 774) \\
\text { units }\end{array}$ & $\begin{array}{c}\text { Significantly reduced LOS } \\
\text { (6.7 vs. } 7.3 \text { days, } p=0.004) \\
\text { Significantly reduced cost } \\
\text { per patient }(\$ 9,477 \mathrm{vs.} \\
\$ 10,451, p<0.001) \\
\end{array}$ & $\begin{array}{l}\text { No significant difference in ADL, } \\
\text { IADL, or mobility performance } \\
\text { at discharge }\end{array}$ & $\begin{array}{l}\text { Gap between time } \\
\text { study conducted } \\
\text { (1993-1997) and } \\
\text { publication (2012) }\end{array}$ & $\begin{array}{c}\text { Randomized } \\
\text { Large sample size }\end{array}$ \\
\hline $\begin{array}{l}\text { Ahmed, et al., } 2012 \text { [19] } \\
\text { (university hospital, USA) }\end{array}$ & Pre/post comparison & $\begin{array}{c}\text { General medical patients } \\
\text { aged } \geq 70 \text { years } \\
\text { (no mean age reported) }\end{array}$ & $\begin{array}{l}\text { Geriatrician or } \\
\text { Geriatric } \\
\text { Consultant with } \\
\text { Private Internist post } \\
\text { (1096) vs. Private and } \\
\text { Teaching Internist } \\
\text { on UC (383) } \\
\text { 1-year pre-ACE }\end{array}$ & $\begin{array}{l}\text { Significant reduction in LOS } \\
(5.55 \text { vs. } 7.76 \text { days; } p=0.001) \\
\text { and CMI adjusted LOS } \\
(5.16 \text { vs. } 6.40 ; p=0.007) \\
\text { year } 2 \text { vs. baseline }\end{array}$ & $\begin{array}{c}\text { No significant difference } \\
\text { in direct costs } \\
\text { Reduced readmission rate from } \\
\text { baseline to years } 1 \text { and } 2 \text { combined } \\
\text { (14.04\% vs. } 11.95 \% \text {; no statistical } \\
\text { analysis performed })\end{array}$ & $\begin{array}{l}\text { Non-randomized } \\
\text { Baseline patients } \\
\text { from multiple } \\
\text { different units } \\
\text { Unequal sample sizes } \\
\text { and time periods in } \\
\text { pre- vs. post-cohorts }\end{array}$ & $\begin{array}{c}\text { Measured CMI } \\
\text { adjusted LOS } \\
\text { Measured direct costs }\end{array}$ \\
\hline $\begin{array}{l}\text { Perez-Zepeda et al., } 2012 \\
\text { [6] (community } \\
\text { hospitals, Mexico) }\end{array}$ & $\begin{array}{l}\text { Prospective } \\
\text { matched cohort }\end{array}$ & $\begin{array}{c}\text { General medical patients } \\
\text { with } \geq 1 \text { targeted } \\
\text { geriatric syndrome } \\
\text { aged } \geq 60 \text { years }(72.6)\end{array}$ & $\begin{array}{l}\text { Geriatricians on ACE } \\
\text { (70) vs. Internist on } \\
\text { UC (140) units }\end{array}$ & $\begin{array}{l}\text { Significantly lower adjusted } \\
\text { OR of composite outcome } \\
\text { (presence of ADL decline, } \\
\text { pressure ulcer, delirium, } \\
\text { or death; } 0.27 \\
95 \% \text { CI } 0.10-0.70 \text { ) }\end{array}$ & $\begin{array}{c}\text { Significantly reduced OR for ADL } \\
\text { decline (0.23; } 95 \% \text { CI } 0.08-0.65) \\
\text { No significant difference in LOS } \\
\text { (9.9 vs. } 9.3 \text { days, } p=\text { NS) } \\
\text { No significant difference in } \\
\text { adjusted OR for hospital mortality } \\
(1.50 ; 95 \% \text { CI } 0.31-7.18)\end{array}$ & $\begin{array}{l}\text { Non-randomized } \\
\text { Small sample sizes for } \\
\text { two-year study } \\
\text { recruitment period }\end{array}$ & $\begin{array}{l}\text { Targeted patients with } \\
\text { existing geriatric } \\
\text { syndromes }\end{array}$ \\
\hline $\begin{array}{c}\text { Flood et al., 2013 [20] } \\
\text { (university hospital, USA) }\end{array}$ & Retrospective cohort & $\begin{array}{l}\text { General medical patients } \\
\text { aged } \geq 70 \text { years }(81.6)\end{array}$ & $\begin{array}{l}\text { Hospitalists on ACE } \\
\text { (428) and UC } \\
\text { (390) units }\end{array}$ & $\begin{array}{c}\text { Significantly reduced } \\
\text { variable direct costs } \\
(\$ 2109 \text { vs. } \$ 2480, p=0.009)\end{array}$ & $\begin{array}{c}\text { Significantly reduced } \\
30 \text {-day readmissions } \\
\text { (7.9\% vs. 12.8\%, } p=0.02) \\
\text { No significant difference in } \\
\text { discharge destination }(p=0.12) \\
\text { including death in hospital } \\
1.4 \% \text { vs. } 1.8 \%)\end{array}$ & $\begin{array}{l}\text { Non-randomized } \\
\text { Data from } \\
\text { administrative } \\
\text { database }\end{array}$ & $\begin{array}{c}\text { Units had same } \\
\text { attendings } \\
\text { Measured variable } \\
\text { direct costs } \\
\text { Reduced costs despite } \\
\text { short LOS }\end{array}$ \\
\hline
\end{tabular}


Table 2. Cont.

\begin{tabular}{|c|c|c|c|c|c|c|c|}
\hline Study (Setting) & Design & $\begin{array}{l}\text { Patient Population (Mean } \\
\text { Age of ACE Cohort) }\end{array}$ & $\begin{array}{c}\text { ACE vs. UC } \\
\text { Attending Physicians } \\
\text { (Sample Sizes) }\end{array}$ & $\begin{array}{c}\text { Primary Outcome } \\
\text { Measured (ACE vs. UC) }\end{array}$ & $\begin{array}{l}\text { Secondary Outcome(s) Measured } \\
\text { (ACE vs. UC) }\end{array}$ & $\begin{array}{l}\text { Study and/or } \\
\text { Intervention } \\
\text { Limitations }\end{array}$ & $\begin{array}{c}\text { Study Strengths } \\
\text { and/or Intervention } \\
\text { Innovations }\end{array}$ \\
\hline $\begin{array}{l}\text { Borenstein, et al., } 2016 \text { [21] } \\
\text { (university hospital, USA) }\end{array}$ & $\begin{array}{l}\text { Cluster RCT of } \\
\text { hospital units }\end{array}$ & $\begin{array}{l}\text { General medical patients } \\
\text { with geriatric risk factors } \\
\text { aged } \geq 65 \text { years }(81.1)\end{array}$ & $\begin{array}{c}\text { Internist on medical } \\
\text { units with (792) and } \\
\text { without (592) ACE } \\
\text { training and } \\
\text { workflow redesign }\end{array}$ & $\begin{array}{l}\text { Observed:Expected LOS } \\
\quad \text { ratio }<1 \text { with ACE } \\
\text { intervention and }>1 \text { on UC }\end{array}$ & $\begin{array}{c}\text { Significantly reduced adjusted OR } \\
\text { of any complication } \\
(0.45,95 \% \text { CI } 0.21-0.98 ; p=0.043) \\
\text { or transfer to ICU } \\
(0.45 ; 95 \% \text { CI } 0.25-0.79 ; p=0.006) \\
\text { Significantly increased adjusted OR } \\
\text { of discharge to PACF } \\
(1.43,95 \% \text { CI } 1.06-1.93 ; p=0.021) \\
\text { No significant difference in } \\
\text { adjusted OR of hospital mortality } \\
(0.69,95 \% \text { CI } 0.42-1.15 ; p=0.16)\end{array}$ & $\begin{array}{l}\text { Non-randomized } \\
\text { Amount of uptake of } \\
\text { NICHE care protocols } \\
\text { on control } \\
\text { units unknown }\end{array}$ & $\begin{array}{l}\text { Large sample size } \\
\text { Redesigned } \\
\text { workflows of all unit } \\
\text { personnel to include } \\
\text { ACE care processes }\end{array}$ \\
\hline $\begin{array}{l}\text { Ekerstad et al., } 2017 \text { [22] } \\
\text { (community } \\
\text { hospital, Sweden) }\end{array}$ & Prospective controlled & $\begin{array}{l}\text { General medical patients } \\
\text { with positive frailty screen } \\
\text { aged } \geq 75 \text { years }(85.7)\end{array}$ & $\begin{array}{l}\text { Internist, Family } \\
\text { Practitioner, and/or } \\
\text { Geriatricians on ACE } \\
\text { (206) vs. Internist on } \\
\text { UC (202) units }\end{array}$ & $\begin{array}{l}\text { Significantly reduced } \\
\text { adjusted OR of decline } \\
\text { in HRQOL } \\
\text { (vision, ambulation, } \\
\text { dexterity, emotion, } \\
\text { cognition, pain dimensions) } \\
3 \text { months post-discharge }\end{array}$ & $\begin{array}{c}\text { Significantly reduced 30-day } \\
\text { readmission } \\
(19 \% \text { vs. } 28 \%, p=0.048) \\
\text { Reduced adjusted 3-month } \\
\text { mortality } \\
\text { (HR 0.55, } 95 \% \text { CI } 0.32-0.96) \\
\text { No significant difference in hospital } \\
\text { mortality ( } 4 \% \text { vs. } 5 \%, p=0.6)\end{array}$ & $\begin{array}{l}\text { Reports trial is } \\
\text { randomized but } \\
\text { patients assigned to } \\
\text { ACE or UC based on } \\
\text { bed availability }\end{array}$ & $\begin{array}{l}\text { Targeted older } \\
\text { frail patients } \\
\text { Evaluated quality } \\
\text { of life }\end{array}$ \\
\hline $\begin{array}{l}\text { Booth et al., } 2018 \text { [23] } \\
\text { (university hospital, USA) }\end{array}$ & Pre/post comparison & $\begin{array}{l}\text { Orthopedic surgery or } \\
\text { medical patients } \\
\text { aged } \geq 65 \text { years }(74.4)\end{array}$ & $\begin{array}{l}\text { Orthopedic Surgeon } \\
\text { or Hospitalist pre (48) } \\
\text { vs. post (113) ACE } \\
\text { workflow redesign }\end{array}$ & $\begin{array}{c}\text { Significantly improved } \\
\text { completion of geriatric } \\
\text { screens for ADL } \\
(62.5 \% \text { vs. } 88.5 \%, p<0.001) \\
\text { and delirium } \\
(4.2 \% \text { vs. } 96.5 \%, p<0.001)\end{array}$ & $\begin{array}{l}\text { Significantly increased patients } \\
\text { mobilized bed to chair } \\
(36.4 \% \text { vs. } 63.5 \%, p<0.05) \\
\text { No significant difference in patients } \\
\text { ambulating in hallway or } \\
\text { delirium prevalence }\end{array}$ & $\begin{array}{l}\text { Non-randomized } \\
\text { Small and unequal } \\
\text { sample sizes/time } \\
\text { periods in cohorts } \\
\text { limits ability to } \\
\text { measure significance }\end{array}$ & $\begin{array}{l}\text { Extension of ACE to } \\
\text { orthopedic } \\
\text { surgery patients } \\
\text { Extension of ACE } \\
\text { without geriatric } \\
\text { specialist }\end{array}$ \\
\hline
\end{tabular}

$\mathrm{RCT}=$ randomized controlled trial; $\mathrm{ACE}=$ Acute Care for Elders; $\mathrm{UC}=$ usual care; $\mathrm{ADL}=$ activities of daily living; $\mathrm{PACF}=$ post-acute care facility; $\mathrm{SW}=$ social work; $\mathrm{PT}=$ physical therapy; $\mathrm{RR}=$ relative risk; $\mathrm{LOS}=$ length of stay; $\mathrm{CHF}=$ congestive heart failure; $\mathrm{UTI}=$ urinary tract infection; $\mathrm{CI}=$ confidence interval; OR = odds ratio; EMR = electronic medical record; $\mathrm{OACE}=$ Oncology-Acute Care for Elders; $\mathrm{BMI}=$ body mass index; IADL = instrumental activities of daily living; CMI = case mix index; NICHE = Nurses Improving Care for Healthsystem Elders; $\mathrm{HRQOL}=$ health related quality of life; $\mathrm{HR}=$ hazard ratio. ${ }^{\alpha}$ global poor outcome measure $=$ death and/or severe ADL dependence and/or poor psychological well-being. * Table modified and reprinted with permission from Malone M, Capezuti E, Palmer R. (eds) Geriatrics Models of Care: Bringing "Best Practice" to an Aging America, copyright Springer Publishing International Switzerland 2015. 
Table 3. ACE service studies and key outcomes *

\begin{tabular}{|c|c|c|c|c|c|c|c|}
\hline Study (Setting) & Design & $\begin{array}{l}\text { Patient Population (Mean } \\
\text { Age of ACE Cohort) }\end{array}$ & $\begin{array}{c}\text { ACE vs. UC } \\
\text { Attending Physicians } \\
\text { (Sample Sizes) }\end{array}$ & $\begin{array}{c}\text { Primary Outcome } \\
\text { Measured } \\
\text { (ACE vs. UC) }\end{array}$ & $\begin{array}{c}\text { Secondary Outcome(s) } \\
\text { Measured } \\
\text { (ACE vs. UC) }\end{array}$ & $\begin{array}{l}\text { Study and/or } \\
\text { Intervention } \\
\text { Limitations }\end{array}$ & $\begin{array}{c}\text { Study Strengths } \\
\text { and/or Intervention } \\
\text { Innovations }\end{array}$ \\
\hline $\begin{array}{c}\text { Wald et al., } 2011 \text { [24] } \\
\text { (university hospital, USA) }\end{array}$ & $\begin{array}{l}\text { Retro-spective chart } \\
\text { review of patients } \\
\text { randomized at time of } \\
\text { admission }\end{array}$ & $\begin{array}{l}\text { General medical patients } \\
\text { aged } \geq 70 \text { years }(80.5)\end{array}$ & $\begin{array}{l}\text { Hospitalist on ACE } \\
\text { (122) vs. Hospitalist or } \\
\text { General or } \\
\text { Subspecialty Internist } \\
\text { on UC (95) services }\end{array}$ & $\begin{array}{l}\text { Significantly increased } \\
\text { patients with documented } \\
\text { recognition and treatment } \\
\text { plan for functional }(68.9 \% \\
\text { vs. } 35.8 \%, p<0.0001) \text { and } \\
\text { cognitive impairment } \\
(55.7 \% \text { vs. } 40 \%, p=0.02) \\
\text { Significantly increased } \\
\text { patients with DNAR orders } \\
(39.3 \% \text { vs. } 26.3 \%, p=0.04)\end{array}$ & $\begin{array}{l}\text { No significant differences } \\
\text { in use of physical } \\
\text { restraints, sleep aids, falls, } \\
\text { discharge location, LOS, } \\
\text { charges, or 30-day } \\
\text { readmissions }\end{array}$ & $\begin{array}{l}\text { Hospitalist-ACE } \\
\text { attendings rotated on } \\
\text { UC services } \\
\text { Intervention did not } \\
\text { include geriatric } \\
\text { training for nurses } \\
\text { LOS and 30-day } \\
\text { readmission rates low } \\
\text { at baseline, limiting } \\
\text { ability to improve } \\
\end{array}$ & $\begin{array}{l}30 \% \text { of ACE patients } \\
\text { located off unit and } \\
\text { received ACE care } \\
\text { Hospitalist-ACE } \\
\text { delivered geriatric } \\
\text { training to residents } \\
\text { Extension of ACE } \\
\text { without geriatric } \\
\text { specialist }\end{array}$ \\
\hline $\begin{array}{c}\text { Yoo et al., } 2013 \text { [25] } \\
\text { (university hospital, USA) }\end{array}$ & $\begin{array}{l}\text { Prospective matched } \\
\text { cohort }\end{array}$ & $\begin{array}{c}\text { Community-dwelling } \\
\text { general medical patients } \\
\text { aged } \geq 65 \text { years } \\
\text { (no mean age reported; } 43 \% \\
\text { of patients aged } \geq 80 \text { ) }\end{array}$ & $\begin{array}{l}\text { Internist on ACE (236) } \\
\text { and UC (248) services }\end{array}$ & $\begin{array}{l}\text { No significant difference } \\
\text { delirium prevalence } \\
(23 \% \text { vs. } 21 \%, p=0.34)\end{array}$ & $\begin{array}{l}\text { Significantly reduced LOS } \\
(6.1 \text { vs. } 6.8 \text { days, } p=0.008) \\
\text { No significant difference } \\
\text { in 30-day readmissions }\end{array}$ & $\begin{array}{l}\text { Non-randomized } \\
\text { Per-protocol and not } \\
\text { intention-to-treat } \\
\text { analysis UC } \\
\text { physicians received } \\
\text { geriatric education }\end{array}$ & $\begin{array}{l}\text { Extension of ACE } \\
\text { without geriatric } \\
\text { specialist }\end{array}$ \\
\hline $\begin{array}{c}\text { Hung et al., 2013 [26] } \\
\text { (university hospital, USA) }\end{array}$ & $\begin{array}{l}\text { Prospective matched } \\
\text { cohort }\end{array}$ & $\begin{array}{l}\text { General medical patients } \\
\text { aged } \geq 75 \text { years }(85.2)\end{array}$ & $\begin{array}{l}\text { Geriatrician on ACE } \\
\text { (173) vs. Internist on } \\
\text { UC (173) services }\end{array}$ & $\begin{array}{c}\text { No significant difference in } \\
30 \text {-day readmissions } \\
(15.4 \% \text { vs. } 22.4 \%, p=0.21) \\
\text { Significantly fewer patients } \\
\text { experiencing an adverse } \\
\text { event (CAUTI, restraint use, } \\
\text { fall, or pressure ulcer; } 9.5 \% \\
\text { vs. } 17.1 \%, p=0.02) \\
\text { Significantly reduced LOS } \\
\text { (4.6 vs. } 6.8 \text { days, } p=0.001)\end{array}$ & $\begin{array}{c}\text { Significantly improved } \\
\text { 3-item CTM mean score } \\
\text { (72.5 vs. } 64.9, p=0.01) \\
\text { No significant difference } \\
\text { in discharge location, } \\
\text { ADL or IADL } \\
\text { performance } 30 \text { days } \\
\text { post-discharge, overall } \\
\text { health status, or HCAHPS } \\
\text { top box satisfaction scores } \\
\text { No significant difference } \\
\text { in } 30 \text {-day mortality } \\
\text { (7.5\% vs. } 5.8 \%, p=0.51)\end{array}$ & $\begin{array}{l}\text { Non-randomized } \\
\text { ACE service only } \\
\text { admitted patients } \\
\text { receiving primary care } \\
\text { in geriatric } \\
\text { patient-centered } \\
\text { medical home }\end{array}$ & $\begin{array}{c}\text { Service includes } \\
\text { geriatrician attending } \\
\text { with allocated social } \\
\text { worker and clinical } \\
\text { nurse specialist }\end{array}$ \\
\hline
\end{tabular}

作

and reprinted with permission from Malone M., Capezuti E., Palmer R. (eds) Geriatrics Models of Care: Bringing "Best Practice" to an Aging America, copyright Springer Publishing

International Switzerland 2015 


\subsection{Studies from ACE Units: Geriatric Processes of Care}

Of the 19 studies examining ACE Units, five examine geriatric care processes and/or recognition of geriatric syndromes as outcome measures; two are RCTs $[9,11,16,17,23]$. Both RCTs found significantly increased documentation of geriatric syndromes in patients admitted to ACE compared to UC units [9,11]. In the Counsell et al., RCT, ACE demonstrated significant increased use of nurse-driven geriatric care protocols and earlier and more frequent consultations to social work and physical therapy.

Two non-randomized studies examined care processes in ACE models that did not require the in-person presence of a geriatric specialist $[16,23]$. Dr. Michael Malone and his team from the Aurora Health Care System have developed ACE Tracker software for use in several electronic medical record (EMR) systems. The ACE Tracker program summarizes in one report geriatric data items documented by various ACE team members (i.e., history of cognitive impairment, results of geriatric screens, presence of potentially inappropriate medications, consultation to social services or rehabilitation therapies). This unit-based ACE Tracker Report is then utilized by an "e-Geriatrician" who can participate in a unit's ACE rounds via conference call using the same electronic ACE Tracker data. In a pre/post study examining geriatric care processes, the e-Geriatrician model demonstrated significant reduction in the use of urinary catheters and increase in consultations for physical therapists on a surgical and medical unit, but no significant change in other processes measured (use of physical restraints, social service assessments, high risk medications) [16].

In a 2018 pilot study, Booth et al., evaluated the impact of a "Virtual ACE" unit intervention that also does not require geriatrician presence either in person or remotely. Team members from all disciplines on a hospital unit are trained in ACE principles, including use of care protocols stemming from standardized nurse assessments for cognitive impairment, delirium, function, and mobility. ACE Tracker capability also exists in this hospital and use of this tool for recognition of geriatric syndromes and interprofessional team communication was included in the training. In pre/post analysis Booth et al., report a significantly increased proportion of patients receiving geriatric screens for function and delirium on two orthopedic surgery units, signaling it may be possible to embed ACE care processes into the routine workflow of non-ACE units throughout a hospital [23].

\subsection{Studies from ACE Units: Function and Mobility Outcomes}

Likely because one of the primary goals of ACE Units was to prevent hospital acquired disability, eleven of the ACE Unit studies evaluated patient performance of activities of daily living (ADL), instrumental activities of daily living (IADL), mobility, and/or discharge to a post-acute care facility, either separately or as part of composite outcome measure $[4,6,7,9,10,12,13,15,18,21,23]$. Six of these studies were randomized and one was a secondary analysis of data collected from a prior RCT $[4,7,9,10,12,18,21]$. The landmark Landefeld et al., study randomized general medical patients age 70 and over to ACE and UC units at a teaching hospital and evaluated function as the primary outcome. Significantly more ACE patients improved performance of ADLs from baseline and admission to time of discharge compared to UC [4]. ACE patients also experienced significantly less post-acute care facility placement $(14 \%$ vs. $22 \%, p=0.01)$. A second RCT from this same investigator group evaluated a new ACE Unit in a community hospital [9]. This study did not demonstrate the same benefits in ADL performance but did demonstrate significant improvement in the composite outcome of ADL decline or need for post-acute care facility placement. The authors noted the logistical challenges in the non-teaching environment due to lack of a physician representative present to participate in the ACE rounds. A third RCT by Barnes et al., also did not demonstrate improvements in function or post-acute care facility referral [18]. Of note, this RCT was conducted on the same ACE unit as in the Landefeld study and the authors noted some of the ACE care processes and environmental redesign were disseminated to UC units during the study period. Finally, a RCT from an ACE Unit in Sweden evaluated a composite outcome measure of death and/or severe ADL dependence and/or poor psychological well-being 3 months after discharge and found no significant difference in ACE versus UC [10]. Another non-USA randomized study of an ACE model for hip fracture patients also 
did not find a significant difference in a composite outcome that included mobility measures 6 months after surgery, except in subgroup analysis of cognitively impaired patients [12].

Several non-randomized studies did report significantly improved ADL performance at time of hospital discharge and/or reductions in post-acute care facility use. Two of these studies came from non-USA-based ACE Units that utilized geriatricians as the attendings $[6,15]$ and one from implementing ACE care principles on a stroke unit [13]. In addition, the previously described Virtual ACE model reported increased patient mobilization [23]. In a study utilizing hospital units as the target for randomization, Borenstein et al. describe a unit-based care redesign intervention similar to the Virtual ACE approach but also included team communication notes with decision support in the EMR. The intervention units were also trained to conduct daily interprofessional team huddles facilitated by an in-person ACE physician advisor who was not directly caring for the patients. Analysis of data, which included patients who screened positive for defined geriatric risk factors, found medical complications were reduced and ACE patients had a significantly increased odds ratio of discharge to a facility [21]. Similar to Barnes et al., the authors noted that ACE care plans were also present to some extent in the comparison units [21].

\subsection{Studies from ACE Units: Additional Outcomes}

Several studies demonstrated other patient outcomes including but not limited to significantly improved self-rated overall health status [4] and health related quality of life [22]; improved patient, caregiver, and provider satisfaction [9]; and reduced complications of care [21]. One study, a RCT targeting older frail patients, evaluated mortality as the primary outcome and reported significantly reduced mortality at 3 and 6 months post-discharge from ACE compared to a UC unit [11]. A more recent study also targeted older frail patients and also noted reduced 3-month mortality [22].

\subsection{Studies from ACE Units: Healthcare Utilization}

In addition to the use of post-acute care facilities as summarized above, fifteen of the unit-based studies reported healthcare utilization (length of stay, readmissions, costs) either as primary or secondary outcome $[6-8,10-16,18-22]$. Ten of these demonstrated either significant reduction or trends toward reduced length of stay (LOS) and/or costs. More recent studies signal the ACE model may reduce costs even in the setting of short LOS. In a 2013 retrospective cohort study of hospitalist patients age 70 and over admitted to ACE versus UC hospitalist units, variable direct costs were significantly reduced despite the fact that both units had a LOS less than 4.3 days [20]. None of the retrieved studies evaluated 30-day readmissions as a primary outcome. Those that included readmissions as a secondary outcome reported either reduced or unchanged readmission rates even when LOS was reduced with ACE team care.

\subsection{Studies from ACE Services}

The literature search yielded three publications describing service-based ACE models (Table 3). In these models, patients admitted to one of these services received ACE-like care even if they were located on different units. The Mobile-ACE (MACE) study specifically states one of the aims is to deliver geriatric care "without the limitations of a physical unit." This model consisted of a geriatrician as the attending with a geriatric fellow, social worker, and clinical nurse specialist assigned to the service rather than a hospital unit. The MACE team conducted daily team meetings addressing geriatric care needs and early care transition planning. This model resulted in significantly reduced adverse events and LOS and an increase in care transitions preparedness as measured by the 3-item the Care Transitions Measure [26,27].

The remaining ACE service studies both utilized an intensive geriatric education experience delivered to general medical teams coupled with implementation of ACE care processes (geriatric screens, care protocols, and interprofessional team meetings). In Wald et al., this approach was implemented with Hospitalists to create the Hospitalist-ACE Service that admitted patients 
preferentially to 12 designated beds but also applied the ACE care and studied the impact in patients on any unit. The team conducted daily (Monday-Friday) 15-min team huddles to coordinate geriatric and care transition plans. This model demonstrated significantly increased recognition of geriatric syndromes and utilization of Do Not Resuscitate orders but did not demonstrate changes in LOS or costs in this program's first year in operation [24]. Yoo et al., implemented the same approach for general medicine services at an academic medical center. The intervention included daily geriatric assessments for function, cognition, delirium, medication reconciliation, and sleep disturbance and three times weekly 45-min interprofessional team meetings. The per-protocol analysis, which only included patients who received at least $80 \%$ of the daily geriatric screens, demonstrated reduced LOS (6.1 days vs. 6.8 days, $p=0.008$ ) [25].

\section{Discussion}

Our review of the twenty-two studies retrieved revealed several themes. The majority of studies are not randomized trials, and those that are occurred predominantly in the first decade of ACE Unit research. One possibility for this limited number of RCTs may be the logistical inability to "hold beds" on designated hospital units due to continually high patient census levels. Non-randomized studies have the associated limitations and therefore any findings and conclusions should be considered within this context. Service related studies are difficult to perform, leading to the disparate study types our search revealed. The lack of standardized and uniform outcome measures or metrics for ACE Units further limits the ability to compare the effectiveness of the various ACE interventions. Many of the ACE studies we found were implementation or feasibility studies and fit into the category of pragmatic trials. Glasgow et al., summarizes the impetus behind the call for increasing the number of pragmatic studies, defined as trials "designed to answer the question of whether a program works under usual conditions, compared to explanatory trials that answer the question if an intervention works under ideal conditions" [28].

We also observed the methodology by which ACE care is implemented has begun to expand. Most commonly, ACE Units continue to care for general medical patients with a unit-based interprofessional team and in-person involvement from a geriatric trained clinician. However, likely in part due to the improved outcomes demonstrated by ACE studies and the increasing numbers of geriatric patients on all units, ACE models are increasingly being deployed for subspecialty populations and operationalized on units and services with and without direct geriatrician participation. We included these studies of ACE care in subspecialty patient populations (stroke, orthopedic surgery) not necessarily for direct comparison to studies of general medical patients, but rather to reveal the dissemination and possible benefits of the ACE model for older adults throughout a hospital.

The mixed results observed in some of the outcomes across studies may be related to the ACE intervention implemented, study design limitations, the outcome measurement tools, or other factors. For example, several studies evaluated outcomes within the first year of operation of a new ACE program. Given the time required to develop high-performing teams in systems accustomed to functioning in silos, repeat evaluation of these models may be beneficial now that time has elapsed. The two studies that demonstrated longer LOS from ACE were both from non-USA hospitals over 15 years ago. Overall the ACE studies in which the usual care LOS was six or more days demonstrated LOS reductions. Cost savings can most easily be attained by reducing LOS. However, ACE Units have also demonstrated reduced costs of care even when LOS is short. This perhaps signals the role of ACE in delivering care that is aligned with patient goals, therefore avoiding unnecessary interventions. Some ACE studies reported increased formal consultation to allied health services such as nutrition and rehabilitation therapies. Another possible future outcome for study may be appropriateness of these referrals, given these resources are often limited and the mandate for stewardship of resources in today's healthcare system. The mixed results in the post-discharge function outcomes measured may signal another opportunity for future intervention and investigation. Additionally, given the barriers of saving beds discussed above, we suspect many ACE Units today admit geriatric patients with 
varied potential for clinical and functional improvement. As one example, the University of Alabama at Birmingham ACE Unit does not utilize any ACE specific admission criteria. This has resulted in an ACE team caring for geriatric patients ranging from fully independent in ADLs to those receiving end-of-life care. Thus, the primary focus of this ACE Unit has become the delivery of "goal-aligned care". This heterogeneous mix of patients with disparate goals will likely require alternative research approaches and measurement tools.

Finally, a common theme in the ACE literature is the prerequisite for training healthcare professionals in the basic principles of geriatric medicine. Given the national shortage of fellowship trained geriatricians, attention to equipping all healthcare providers with this knowledge is essential to disseminating ACE care and achieving the ultimate goal to establish Age-Friendly Health Systems [29]. National institutions and funding mechanisms are required to support this education. Examples of such support include the Nurses Improving Care for Healthsystem Elders institution [30] and the Health Resources and Services Administration funded Geriatric Workforce Enhancement Programs [31]. The basic training of all healthcare providers in geriatrics could then shift the role of fellowship trained geriatricians to lead more system-level initiatives to further promote age-friendly care delivery [32]. Extending beyond geriatric education, working in teams has been recognized as a mechanism for improving quality and care coordination for patients of all ages. Future opportunities may also include merging patient safety teamwork curriculum such as TeamSTEPPS ${ }^{\circledR}$ (Team Strategies \& Tools to Enhance Performance \& Patient Safety) with ACE team training. Available from the Agency for Healthcare Research and Quality [33], TeamSTEPPS ${ }^{\circledR}$ use has resulted in sustained improvements in patient safety [34]. In addition to the challenges of providing geriatric education to all healthcare providers, other barriers hospitals face in developing ACE Units include lack of hospital-based geriatric providers with protected time and effort to lead program development, challenges in securing dedicated unit space, and the need for some start-up funding, despite published evidence of return on this investment. While dedicated ACE Units will likely remain the core model, the future for ACE care, and a means to address development barriers, may reside in the current trend toward embedding ACE care processes into existing hospital systems and workflows.

\section{Conclusions}

The patient characteristics and outcome measures in these 22 ACE studies are varied. While we have summarized key variables that may influence outcomes (i.e., mean age, mortality rates), conducting an in-depth systematic review and meta-analysis is beyond the scope of this literature overview. Rather, we conducted this overview with the goal to concisely summarize ACE studies to serve as a reference tool for clinicians and researchers working to improve and expand the implementation of team care for all hospitalized elders. Despite our attempt to be thorough in our search we suspect there are additional ACE unit studies that were not retrieved with our strategy. We also did not conduct an in-depth rating of the quality of each study retrieved. Overall, the majority of the studies demonstrated improved quality with simultaneous reductions in measures of inefficiency (LOS) that were cost-neutral or cost-saving. These outcomes represent value-based care and are aligned with the goals the IOM called for almost two decades ago.

Funding: This research received no external funding.

Acknowledgments: We thank Sara N. Biswal for her assistance with revising our manuscript. We thank the entire UAB ACE Unit interprofessional team and UAB Hospital leadership for their dedication to providing the highest quality of care to all of our patients. Finally, we thank our patients and families for giving us the privilege of participating in their care and for assisting us in our ongoing efforts to improve.

Conflicts of Interest: The authors declare no conflict of interest. 


\section{References}

1. Institute of Medicine Committee on Quality of Health Care in America. To Err is Human: Building a Safer Health System; Kohn, L.T., Corrigan, J.M., Donaldson, M.S., Eds.; National Academies Press (US): Washington, DC, USA, 2000.

2. Institute of Medicine Committee on Quality of Health Care in America. Crossing the Quality Chasm: A New Health System for the 21st Century; National Academies Press (US): Washington, DC, USA, 2001.

3. Palmer, R.M.; Landefeld, C.S.; Kresevic, D.; Kowal, J. A medical unit for the acute care of the elderly. J. Am. Geriatr. Soc. 1994, 42, 545-552. [CrossRef] [PubMed]

4. Landefeld, C.S.; Palmer, R.M.; Kresevic, D.M.; Fortinsky, R.H.; Kowal, J. A randomized trial of care in a hospital medical unit especially designed to improve the functional outcomes of acutely ill older patients. N. Engl. J. Med. 1995, 332, 1338-1344. [CrossRef] [PubMed]

5. Grant, M.J.; Booth, A. A typology of reviews: An analysis of 14 review types and associated methodologies. Health Inf. Libr. J. 2009, 26, 91-108. [CrossRef] [PubMed]

6. Perez-Zepeda, M.U.; Gutierez-Robledo, L.M.; Sanchez-Garcia, S.; Juarez-Cedillo, T.; Gonzalez, J.J.; Franco-Marina, F.; Garcia-Pena, C. Comparison of a geriatric unit with a general ward in Mexican elders. Arch. Gerontol. Geriatr. 2012, 54, e370-e375. [CrossRef] [PubMed]

7. Covinsky, K.E.; King, J.T., Jr.; Quinn, L.M.; Siddique, R.; Palmer, R.; Kresevic, D.M.; Fortinsky, R.H.; Kowal, J.; Landefeld, C.S. Do acute care for elders units increase hospital costs? A cost analysis using the hospital perspective. J. Am. Geriatr. Soc. 1997, 45, 729-734. [CrossRef] [PubMed]

8. Stewart, M.; Suchak, N.; Scheve, A.; Popat-Thakkar, V.; David, E.; Laquinte, J.; Gloth, F.M., 3rd. The impact of a geriatrics evaluation and management unit compared to standard care in a community teaching hospital. Md. Med. J. 1999, 48, 62-67. [PubMed]

9. Counsell, S.R.; Holder, C.M.; Liebenauer, L.L.; Palmer, R.M.; Fortinsky, R.H.; Kresevic, D.M.; Quinn, L.M.; Allen, K.R.; Covinsky, K.E.; Landefeld, C.S. Effects of a multicomponent intervention on functional outcomes and process of care in hospitalized older patients: A randomized controlled trial of Acute Care for Elders (ACE) in a community hospital. J. Am. Geriatr. Soc. 2000, 48, 1572-1581. [CrossRef] [PubMed]

10. Asplund, K.; Gustafson, Y.; Jacobsson, C.; Bucht, G.; Wahlin, A.; Peterson, J.; Blom, J.O.; Angquist, K.A. Geriatric-based versus general wards for older acute medical patients: A randomized comparison of outcomes and use of resources. J. Am. Geriatr. Soc. 2000, 48, 1381-1388. [CrossRef] [PubMed]

11. Saltvedt, I.; Mo, E.S.; Fayers, P.; Kaasa, S.; Sletvold, O. Reduced mortality in treating acutely sick, frail older patients in a geriatric evaluation and management unit. A prospective randomized trial. J. Am. Geriatr. Soc. 2002, 50, 792-798. [CrossRef] [PubMed]

12. Naglie, G.; Tansey, C.; Kirkland, J.L.; Ogilvie-Harris, D.J.; Detsky, A.S.; Etchells, E.; Tomlinson, G.; O'Rourke, K.; Goldlist, B. Interdisciplinary inpatient care for elderly people with hip fracture: A randomized controlled trial. CMAJ 2002, 167, 25-32.

13. Allen, K.R.; Hazelett, S.E.; Palmer, R.R.; Jarjoura, D.G.; Wickstrom, G.C.; Weinhardt, J.A.; Lada, R.; Holder, C.M.; Counsell, S.R. Developing a stroke unit using the acute care for elders intervention and model of care. J. Am. Geriatr. Soc. 2003, 51, 1660-1667. [CrossRef] [PubMed]

14. Jayadevappa, R.; Chhatre, S.; Weiner, M.; Raziano, D.B. Health resource utilization and medical care cost of acute care elderly unit patients. Value Health 2006, 9, 186-192. [CrossRef] [PubMed]

15. Zelada, M.A.; Salinas, R.; Baztan, J.J. Reduction of functional deterioration during hospitalization in an acute geriatric unit. Arch. Gerontol. Geriatr. 2009, 48, 35-39. [CrossRef] [PubMed]

16. Malone, M.L.; Vollbrecht, M.; Stephenson, J.; Burke, L.; Pagel, P.; Goodwin, J.S. AcuteCare for Elders (ACE) tracker and e-Geriatrician: Methods to disseminate ACE concepts to hospitals with no geriatricians on staff. J. Am. Geriatr. Soc. 2010, 58, 161-167. [CrossRef] [PubMed]

17. Flood, K.L.; Brown, C.J.; Carroll, M.B.; Locher, J.L. Nutritional processes of care for older adults admitted to an oncology-acute care for elders unit. Crit. Rev. Oncol. Hematol. 2011, 78, 73-78. [CrossRef] [PubMed]

18. Barnes, D.E.; Palmer, R.M.; Kresevic, D.M.; Fortinksy, R.H.; Kowal, J.; Chren, M.-M.; Landefeld, C.S. Acute Care For Elders Units Produced Shorter Hospital Stays At Lower Cost While Maintaining Patients' Functional Status. Health Aff. 2012, 31, 1227-1236. [CrossRef] [PubMed] 
19. Ahmed, N.; Taylor, K.; McDaniel, Y.; Dyer, C.B. The role of an Acute Care for the Elderly unit in achieving hospital quality indicators while caring for frail hospitalized elders. Popul. Health Manag. 2012, 15, 236-240. [CrossRef] [PubMed]

20. Flood, K.L.; Maclennan, P.A.; McGrew, D.; Green, D.; Dodd, C.; Brown, C.J. Effects of an acute care for elders unit on costs and 30-day readmissions. JAMA Intern. Med. 2013, 173, 981-987. [CrossRef] [PubMed]

21. Borenstein, J.E.; Aronow, H.U.; Bolton, L.B.; Dimalanta, M.I.; Chan, E.; Palmer, K.; Zhang, X.; Rosen, B.; Braunstein, G.D. Identification and team-based interprofessional management of hospitalized vulnerable older adults. Nurs. Outlook 2016, 64, 137-145. [CrossRef] [PubMed]

22. Ekerstad, N.; Karlson, B.W.; Dahlin Ivanoff, S.; Landahl, S.; Andersson, D.; Heintz, E.; Husberg, M.; Alwin, J. Is the acute care of frail elderly patients in a comprehensive geriatric assessment unit superior to conventional acute medical care? Clin. Interv. Aging 2017, 12, 1-9. [CrossRef] [PubMed]

23. Booth, K.A.; Simmons, E.E.; Viles, A.F.; Gray, W.A.; Kennedy, K.R.; Biswal, S.H.; Lowe, J.A.; Xhaja, A.; Kennedy, R.E.; Brown, C.J.; et al. Improving Geriatric Care Processes on Two Medical-Surgical Acute Care Units: A Pilot Study. J. Healthc. Qual. Off. Publ. Natl. Assoc. Healthc. Qual. 2018. [CrossRef] [PubMed]

24. Wald, H.L.; Glasheen, J.J.; Guerrasio, J.; Youngwerth, J.M.; Cumbler, E.U. Evaluation of a hospitalist-run acute care for the elderly service. J. Hosp. Med. 2011, 6, 313-321. [CrossRef] [PubMed]

25. Yoo, J.W.; Kim, S.; Seol, H.; Kim, S.J.; Yang, J.M.; Ryu, W.S.; Min, T.J.; Choi, J.B.; Kwon, M.; Nakagawa, S. Effects of an internal medicine floor interdisciplinary team on hospital and clinical outcomes of seniors with acute medical illness. Geriatr. Gerontol. Int. 2013, 13, 942-948. [CrossRef] [PubMed]

26. Hung, W.W.; Ross, J.S.; Farber, J.; Siu, A.L. Evaluation of the Mobile Acute Care of the Elderly (MACE) service. JAMA Intern. Med. 2013, 173, 990-996. [CrossRef] [PubMed]

27. Coleman, E.A.; Smith, J.D.; Frank, J.C.; Eilertsen, T.B.; Thaire, J.N.; Kramer, A.M. Development and testing of a measure designated to assess the quality of care transitions. Int. J. Integr. Care 2002, 2, e02. [CrossRef] [PubMed]

28. Glasgow, R.E. What does it mean to be pragmatic? Pragmatic methods, measures, and models to facilitate research translation. Health Educ. Behav. 2013, 40, 257-265. [CrossRef] [PubMed]

29. Fulmer, T.; Mate, K.S.; Berman, A. The age-friendly health system imperative. J. Am. Geriatr. Soc. 2018, 66, 22-24. [CrossRef] [PubMed]

30. Nurses Improving Care for Healthsystem Elders (NICHE). Available online: https://nicheprogram.org (accessed on 28 June 2018).

31. Health Resources \& Services Administration. Geriatrics Workforce Enhancement Program. Available online: https:/ / bhw.hrsa.gov / fundingopportunities / default.aspx?id=9f260dcc-0978-4c96-8a57-e0a767840ef0w (accessed on 28 June 2018).

32. Callahan, K.E.; Tumosa, N.; Leipzig, R.M. Big ' $G$ ' and Little 'g' Geriatrics Education for Physicians. J. Am. Geriatr. Soc. 2017, 65, 2313-2317. [CrossRef] [PubMed]

33. Agency for Healthcare Research and Quality RM. TeamSTEPPS 2.0. Web Site. Available online: https: / / www.ahrq.gov/teamstepps/instructor/index.html (accessed on 25 March 2018).

34. Rasin-Waters, D.; Abel, V.; Kearney, L.K.; Zeiss, A. The integrated care team approach of the Integrated of the Department of Veterans Affairs (VA): Geriatric Primary Care. Arch. Clin. Neuropsychol. 2018, 33, 280-289. [CrossRef] [PubMed]

(C) 2018 by the authors. Licensee MDPI, Basel, Switzerland. This article is an open access article distributed under the terms and conditions of the Creative Commons Attribution (CC BY) license (http://creativecommons.org/licenses/by/4.0/). 